\title{
Bipolar and Substance Abuse: The Dual-Diagnosis Client
}

Kimberly Scarcelli and LaVelle Hendricks*

Department of Psychology and Counseling, Texas A\&M University Commerce, USA

\begin{abstract}
The concept of a client having both a Substance Abuse Disorder (SAD) as well as a mental disorder is becoming more prevalent with 25 to $33 \%$ of all mental health patients considered dual-diagnosed [1]. While counselors are equipped to address one of the disorders in clients readily, the comorbidity creates difficulty in best meeting the client's needs. The combination of Bipolar and Substance Abuse Disorder in an individual creates two key focal areas to address in counseling that may actually conflict with the other, or in some cases, one disorder may instigate the other. A need exists for both assessments and treatments that address this comorbidity combination.

The concept of a client having both a substance abuse disorder as well as a mental disorder becoming more prevalent with 25 to $33 \%$ of all mental health patients considered dual-diagnosed [1]. The psychiatric textbooks based their findings on little data and clinical lore [2]. Soon after, epidemiological studies were done on both substance abuse clients as well as those with mental disorders. The studies determined that not only was dual-diagnosis more prevalent, but also that a large number of those that have a mental disorder also have issues with substance use [2] There are many disorders that can be combined with those that suffer from a substance use disorder (SUD) including eating disorders, gambling, compulsive shopping, and sexual disorders, as well has other physical and psychological disorders. In this paper, the disorders of bipolar and substance abuse will be identified, but also, attention will be given to the comorbid client that suffers from both disorders and potential treatment for this individual.
\end{abstract}

Keywords: Bi-polar; Substance; Abuse; Disorder

\section{The Bipolar-Diagnosed Client}

Bipolar disorder is a group of "chronically recurring disorders of varying and fluctuating intensity, characterized by intermittent or prolonged affective instability, states of inhibition and excitement of various mental functions such as mood, cognition and psychomotricity" [3]. Typically, the bipolar client will shift between manic and depressive stages, often occurring in situations where the opposite reaction would have been the norm. For each client, the "mood swings [will] vary in severity, duration, and sequence" [4]. Though relatively common, bipolar disorder is severe and persistent. The illness affects $1.3-1.7 \%$ of the population [5]. In an early study "[i]n the 1920s, Kraepelin recognized the frequent occurrence of alcoholism in manic-depressives" [6]. It was determined that persons with Bipolar I disorder had a $46 \%$ chance that an alcohol-related disorder would be prevalent in their life compared to the $14 \%$ of those with alcohol-based disorders in the entire population [7].

\section{The Substance Abuse Diagnosed Client}

People have had issues with various types of substance abuse for thousands of years. Since the 2000s, this disorder has been most widespread of all the disorders in mental health [2]. With society constantly changing what is considered more correct to say, the Diagnostic and Statistical Manual of Mental Disorders, Fifth Edition (DSM-5), stopped using the terms of substance abuse and dependence. It now calls the disorder, substance use disorder, which is defined as "mild, moderate, or severe to indicate the level of severity, which is determined by the number of diagnostic criteria met by an individual" [8]. An addiction is a disorder that spreads by extended exposure to an internally addictive substance. "As a disease, addiction essentially impairs the individual's capability to achieve any subjectively pleasant equilibrium, while his or her pleasure-seeking behavior grows to a counterproductive, dysfunctional level. Far from being just a deviant lifestyle pattern, or a rewarding 'personal choice' for deviance, addiction never provides a balance, whether it is a deviant or more extreme antisocial type" [3]. There are many types of substance use disorders (SUDs) including those that are alcohol or tobacco-based. "The most prominent form of SUDs is the abuse of illegal drugs such as hallucinogens, cocaine, narcotics, and marijuana" [2]. Like most things, substance abuse is a trend of what is considered popular at the moment. Popularity often is based upon accessibility, location, and cost. Regardless, addiction to these chemicals is an on-going issue in the United States and other countries. "When all the direct and indirect costs of substance abuse and addiction are added up, it has been found that over $15 \%$ of the average state's budget is devoted to the costs of substance abuse disorders" [2]. Other statistics stated by Doweiko are that:

- About one-fourth of those seen by a general physician have some type of substance abuse disorder.

- Half of those that died due to extreme trauma-based injury were due to too much alcohol consumed.

- Over $40 \%$ of hospital inpatients have some issue relating to alcohol.

- As of 2008, of Medicaid recipients, one-fourth of them have a substance abuse issue.

Additionally, for Americans over the age of 12, there are approximately 22.2 million that have a type of SUD [9]. According to

*Corresponding author: LaVelle Hendricks, Department of Psychology and Counseling, Texas A\&M University Commerce, USA, Tel: 9038865652; E-mail: Lavelle.Hendricks@tamuc.edu

Received August 07, 2017; Accepted September 05, 2017; Published September 08, 2017

Citation: Scarcelli K, Hendricks LV (2017) Bipolar and Substance Abuse: The Dual-Diagnosis Client. J Health Educ Res Dev 5: 228. doi: 10.4172/23805439.1000228

Copyright: $\odot 2017$ Scarcelli K, et al. This is an open-access article distributed under the terms of the Creative Commons Attribution License, which permits unrestricted use, distribution, and reproduction in any medium, provided the original author and source are credited. 
Van Wormer and Davis, at least two of the following must take place within a year for a client to be considered as having an SUD [10]:

- While using substances, a pattern of not completing responsibilities that come with work, school, or home.

- During substance use, placing him/her in dangerous scenarios.

- While using substances, problems occurring in relationships.

- Tolerance of the substance through increased need or less side effects in use.

- Withdrawal symptoms or the need to take the drug to relieve said symptoms.

- Increase in use or amount taken over time.

- The ineffectual desire to stop taking or lessen the intake of the substance.

- Marked time given to the gathering, using, or quitting substances.

- Primary activities have lessened or stopped altogether due to usage.

- The continuation of substance use despite the knowledge of the ramifications.

- An on-going craving for the substance.

Substances can fall into seven main categories which include: depressants, stimulants, hallucinogens, narcotics, inhalants, cannabis, and dissociative drugs. Depressants tend to slow down how the body and brain operate. They include alcohol, barbiturates, and antianxiety tranquilizers. Stimulants will stimulate heart rate and cause blood pressure to rise. Examples include cocaine and amphetamines. Hallucinogens alter the user's visual perception. The most common of hallucinogens is LSD. Narcotics are often used to lessen pain and alter mood. Common narcotics are opium, codeine, heroin, morphine, and OxyContin. Inhalants are substances which are inhaled or breathed-in that will produce side effect that alter mental results and effects. Cannabis, often known as marijuana produces a euphoric, but temporary high. Dissociative drugs stop the message line from the brain to an area of pain. PCP is a well-known dissociative drug [2].

\section{The Dual-Dependent Diagnosed Client}

When an addiction, whether substance or behavioral is coupled to one with a mental disorder, the client would be considered a dualdiagnosed client. "Behavioral addictions (BAs) can be understood as disorders characterized by repetitive occurrence of impulsive and uncontrolled behaviors that cause psychological, social and working problems, sometimes also legal and economic consequences [11]. Features common with these addictions include:

- The inability to resist impulses

- The urge to fulfill an action that would be harmful to oneself or another

- The increasing enthusiasm or eagerness about accomplishing the action

- Immense pleasure or euphoria at the time of the action [11].

It is still being determined if behavioral addictions are connected to either mood or substance use disorders [11]. Many like to differentiate between the two separate diagnoses, however, the symptoms and syndromes often overlap feeding off of each other. "This makes dual diagnosis a special subtype of "comorbidity" as it is customarily used in current medical and psychiatric literature" [4]. While there are two very diverse subcomponents within the individual; "nonetheless they do share a common anatomic and physiologic ground in the central nervous system, with the result that mutual interaction between them is not just an additional clinical issue, but the crucial foundation on which to build a combined pathophysiological model and related treatment approaches" [4]. When a client has both an addiction and a mental disorder, the mixing of the two together often is more destructive to the person. "Different psychiatric conditions may have different impacts on substance use; they may involve different interactions with substances, and have a different relationship with self-administration practices" [4]. For many, self-medication becomes an issue. Those that are addicted to a substance and use it as a way to self-medicate often are categorized as "substance-abusing mentally ill patients" [4]. This process of self-medication is often used as a way to cope with the mental disorder. However, typically, these same individuals not only will medicate as relief, but they will also continue using when the relief is no longer there.

According to Regier et al. [7] the highest rate of co-occurrence of a mental disorder combined with a substance use disorder is found among clients diagnosed with bipolar disorder. "The National Epidemiological Catchment Area Study (ECA) found a 56\% lifetime prevalence of substance abuse or dependence among persons with bipolar disorder" [12]. According to Doweiko [2], of those diagnosed with bipolar disorder, between 40 and $70 \%$ will also portray evidence of SUD. Those patients with this dual-diagnosis often show characteristics of lack of insight, lower cognitive skills, high suicide rate, denial, and a greater chance of not adhering to the treatment subscribed. Those with both bipolar and substance abuse disorders often have multiple relapses with substances. It is believed that "[i]n turn, concurrent substance disorder increases the chronicity, disability, and mortality of bipolar disorder" [12]. Anxiety issues are often three times more likely for these patients. "In a sub-analysis of 33 patients with bipolar disorder, they found bipolar patients with panic attacks were more likely to have disorders of cocaine use, sedative use, and stimulant use compared to bipolar patients without panic attacks [12]. Also seen in these clients during the manic stage of the bipolar, is a heightened sense of overconfidence [2]. According to Weiss [13], those who are dually diagnosed with both substance use and bipolar disorders usually have worse outcomes that with just one of the two. They also have a great chance of medication noncompliance; moods will be longer lasting with slower recovery, most hospitalization time, and higher suicide rates [13]. Drug usage tends to increase during the manic stage of the bipolar cycle. Those with an alcohol dependency seem to increase usage more during the depression stage [2].

\section{Counseling/Advising the Dual-Dependent Bipolar- Substance Abuser}

Although it has been proven that the dual-diagnosed bipolar patient has a great probability of also having a substance abuse issue, very little has been developed as far as treatment for these clients. Because there is not any data on this treatment, counselors must make assessments based upon clinical data. Some believe that treatment for those with dual disorders would be beneficial if both disorders were treated concurrently [14]. Another issue is that "it remains unclear whether first-line medication strategies and algorithms developed for bipolar and bipolar spectrum disorders are appropriate for patients with comorbid substance use disorders" [14].

Those that are currently researching the bipolar-substance 
abuser are using both medication as well as psychotherapy in their treatments. In clinical, these methods are both being done singularly as well in conjunction with each other. There are several reasons why both methods may be used in counseling. "First, substance use disorders often go unrecognized or are viewed as a secondary problem in psychiatric treatment settings. Second, substance abuse treatment settings may misconstrue irritability or 'acting-out' behavior as secondary to substance use rather than underlying affective symptoms. In both settings, treatment providers may not have the expertise to treat both disorders or the program may receive financial reimbursement for treating only one of the disorders" [14]. The main areas of treatment include, "assertive outreach, comprehensive services, and flexible as well as a stage wise treatment incorporating motivational strategies to increase the patient's readiness for abstinence-based treatment approaches" [15]. Integrated treatment has had positive results reducing the time of hospital stay as well as higher life satisfaction. "In subsequent, longer-term uncontrolled studies of integrated treatment (18 months to 4 years), improvements in substance abuse outcomes were found, suggesting that the benefits of integrative interventions for severely mentally ill substance abusers may require an extended period to 'take hold"' [14].

While other treatment has been used successfully, group therapy was limited in the results. Many studies attribute this to the fact that many bipolar diagnosed clients also have additionally psychotic disorders such as schizophrenia. Treatment as an integrated group would need individual treatment plans within to meet the special needs. According to Weiss [13] in an Integrated Group Therapy (IGT) designed to test clients that have a dual-diagnosis of bipolar and substance abuse disorders, there were five key themes within the treatment.

- The "Single Disorder Paradigm" in which either client or counselor place the client into only one of the disorders. To dissuade from the usage of a singular disorder, the term "bipolar substance abuse" was created.

- The majority of the clients with this comorbidity have a strong inclination towards depression. It was common in this study for patients to be more medicinally compliant over the fear of the depressive state rather than the manic.

- Between the two disorders, many parallels occurred. Both thoughts and behaviors were reflected.

- The two disorders would react from each other.

- These clients showed different depths of hopelessness as they cycled both through the bipolar or treatments.

The bipolar substance abuse client is very likely to not be medicinally compliant. It is common for the individual to seek more substances in order to self-medicate rather than take the prescribed medication. Due to this, the counselor would need to reiterate the damage that substance abuse could have on the client.

Within data still relatively scarce, the counselor will need to be aware of specific needs within the client. The counselor will also need to be open to outside resources and thinking to best meet the needs of each. While bipolar and substance abuse comorbidity is not specific to any one multicultural group, being cognizant of needs to specific cultures would only benefit both counselor and client in the end.

\section{Obstacles Treating the Dual-Diagnosed Bipolar Client}

One of the first obstacles faced for counseling the comorbid client stems from the mood swings encountered in bipolar. There is an increased use of substances during the manic stage rather than the depressive. "The trend toward a higher rate of drug abuse during mania than depression in bipolar patients, and higher overall rates I bipolar than unipolar patients reported, suggest mania may be more associated with drug use than depression" [5]. There are higher rates of suicidal attempts with the dual-diagnosed patient who started the substance abuse after the start of the affective illness [5].

Maremmani [3] discuss how the comorbidity had a direct correlation to concomitant use of cocaine. The cocaine use became predominant during the stages of social adjustment. While family issues did not cause a reaction, problems in the work environment or legal issues did. "Regarding substance abuse history, concomitant use of cocaine in heroin addicts correlated with patterns of heroin use (a history of failed treatments, the necessity to associate more than one treatment (methadone treatment and other psychoactive substances and/or psychotherapy and/or residential program), younger age of first substance use, younger age of continuous use and major dependence duration" [4]. If the manic episode was triggered through substance use, full-blown bipolar may manifest with additional psychotic symptoms.

With two obvious disorders, there is confusion on how to best assess the client. "Diagnostic confusion is less likely to occur for individuals with chronic, severe bipolar disorder or those who clearly developed bipolar symptoms before substance abuse" [14]. The Diagnostic and Statistics Manual of Mental Disorders DSM-IV-TR mentions different approaches on how to clarify the diagnostic data. The first is the determination of when the bipolar symptoms began, before or after the start of using substances. Secondly, assessing whether the symptoms for bipolar lasted long after the use of substances. Third, the counselor should determine if the symptoms are beyond the norm for bipolar [14].

There is an overrepresentation of substance use disorders in bipolar disorder clients. There are three possible reasons for this: selfmedication, substance abuse may cause bipolar, and bipolar may cause substance abuse [14]. The qualitative procedures used was to study the literature and find the various approaches used in determining the link between substance use disorders and bi polar disorders. The first is when there is an attempt to counteract the bipolar symptoms through the use of non-prescriptive medication. The second is based on the results of different studies that discovered that the abuse of substances could potentially lead to bipolar. Levin and Hennessy (2004) state that in a study three-fourths of 77 hospitalized patients with early staged bipolar had a substance abuse disorder at least a year prior to the first bipolar manic episode. The third reason disagrees with the prior believing that the bipolar symptoms triggered the substance use and later abuse. This was based on the fact that manic stages can be documented in bipolar clients who were diagnosed as young children who have not yet encountered substances, but later in life were diagnosed as having substance abuse disorder as well. The bipolar disorder often reverts to the substance use and abuse due to the impulsivity, poor judgement, and the over-abundance of interaction found in pleasurable activities associated with the usage. By definition, "[i]mpulsivity can be conceptualized as a predisposition towards rapid, unplanned actions without regard to consequences" [16,17]. Within bipolar, impulsivity is one of the criteria found in the manic cycle when diagnosing.

There are negative results on those that have bipolar regarding the side effects of substance abuse. For starters, the two combined may result in poor treatment outcome. A study was conducted "examining 256 acutely manic patients evaluated in an urban psychiatric facility 
and found that acutely manic patients with current alcohol misuse presented with a higher total number of manic symptoms as well as increased mood liability, impulsivity, and violence than did acutely manic patients without current alcohol misuse [14].

Over time, it has become more apparent that the counseling of the dual-diagnosed bipolar-substance abuse client is diverse with each client. Each client has a cycle unique to themselves. Some lean more towards the manic stage with extreme violence, while other is more depressive and lethargic. When counseling these unique individuals, it is paramount to not generalize either condition or treatment.

\section{Conclusion}

It is apparent, that drug or substance abuse added to diagnosed bipolar clients is a significant health issue. Data supports that there is an extremely high rate of substance use and abuse among the manicdepressive person. Awareness of the prevalence of drug use in the bipolar client is necessary for effective evaluation. While it is widely recognized that these two disorders are often found together, there still remains little data to assist in which treatment types would be most beneficial. "Because this patient population is at such risk for untoward events (i.e., hospitalizations and suicides), it is urgent that treatments be developed and tested in this challenging patient population" [14].

\section{References}

1. Frequently Asked Questions (2017) Behavioral Health Evolution.

2. Doweiko HE (2012) Concepts of Chemical Dependency. 8th edn. Belmont, CA, USA. Brooks/Cole.

3. Maremmani I, Perugi G, Pacini M, Akiskal HS (2006) Toward a unitary perspective on the bipolar spectrum and substance abuse: Opiate addiction as a paradigm. Journal of Affective Disorders 93: 1-12.

4. Maremmani I, Pacini M, Perugi G, Deltito J, Akiskal H, et al. (2008) Cocaine abuse and the bipolar spectrum in 1090 heroin addicts: Clinical observations and a proposed pathophysiologic model. Journal of Affective Disorders 106: 55-61.
5. Brown ES, Suppes T, Adinoff B, Thomas NR (2001) Drug abuse and bipolar disorder: Comorbidity or misdiagnosis? Journal of Affective Disorders 65: 108115.

6. Goodwin FK, Jamison KR (1990) Alcohol and drug abuse in manic-depressive illness. In: Manic-Depressive Illness. Oxford, pp: 210-226.

7. Regier DA, Farmer ME, Rae DS, Locke BZ, Keith SJ, et al. (1990) Comorbidity of mental disorders with alcohol and other drug abuse. J Am Med Assoc 264 2511-2518.

8. Enomoto K (2015) SAMHSA (United States, Substance Use and Mental Health Services Administration).

9. Nomamiukor N, Brown ES (2009) Attrition factors in clinical trials of comorbid bipolar and substance-related disorders. Journal of Affective Disorders 112 284-288.

10. Van Wormer K, Davis DR (2013) Addiction Treatment: A Strength's Perspective. Belmont, CA. Brooks/Cole, Cengage Learning. 3rd edn.

11. Di Nicola M, Tedeschi D, Mazza M, Martinotti G, Harnic D, et al. (2010) Behavioural addictions in bipolar disorder patients: Role of impulsivity and personality disorders. Journal of Affective Disorders 125: 82-88.

12. Mitchell JD, Brown ES, Rush AJ (2007) Comorbid disorders in patients with bipolar disorder and concomitant substance dependence. Journal of Affective Disorders 102: 281-287.

13. Weiss RD (2004) Treating patients with bipolar disease and substance dependence: Lessons learned. Journal of Affective Disorders 27: 307-312.

14. Levin FR, Hennessy G (2004) Bipolar disorder and substance abuse. Biological Psychology 56: 738-748.

15. Drake RE, Mueser KT, Clark RE, Wallach MA (1996) The course, treatment and outcome of substance disorder in persons with severe mental illness. Am J Orthopsychiatry 66: 42-51.

16. Lewis M, Scott J, Frangou S (2009) Impulsivity, personality and bipolar disorder. European Psychiatry 24: 464-469.

17. Weiss RD, Kolodziej M, Griffin ML, Najavits LM, Jacobson LM, et al. (2004) Substance use and perceived symptom improvement among patients with bipolar disorder and substance dependence. Journal of Affective Disorders 79 279-283. 